Recepción: 15/06/2014

Evaluación: 28/08/2014

Aprobación: 20/10/2014

Artículo de Reflexión

\title{
UNIVERSIDAD: DEL CAPITAL HUMANO A UN MODELO DE EMPRESA EN LA EDUCACIÓN SUPERIOR ${ }^{1}$
}

\author{
Álvaro Acevedo Tarazona ${ }^{2}$ \\ Universidad Industrial de Santander \\ Grupo de Investigación: Políticas, \\ Sociabilidades y Representaciones Histórico-Educativas \\ tarazona20@gmail.com
}

\begin{abstract}
RESUMEN
Este artículo es una reflexión acerca del papel de la Universidad en la sociedad y sobre su cambiante configuración, hasta convertirse en una empresa. Al modelo universitario norteamericano, imperante durante los años sesenta y setenta, basado en la técnica y el conocimiento científico, lo está reemplazando un concepto neoliberal que ha hecho de la Educación Superior una unidad empresarial a partir de las lógicas del mercado. En este debate, la universidad se ha planteado desde dos orillas: en su función de empresa o de conocimiento, lo que lleva a preguntarse por los temas educativos de lo superior, sin resolución del continente, y por los enfoques locales y nacionales.
\end{abstract}

Palabras clave: Universidad, investigación, conocimiento, empresa, capital humano.

1. Este texto expone algunos de los resultados de investigación obtenidos en los proyectos: La experiencia histórica del cogobierno en la Universidad Industrial de Santander: concepciones y divergencias en disputa por la autonomía universitaria, 1971-1976 y el proyecto Desenvolvimiento histórico de la Escuela de Artes y Oficios de Bucaramanga: primer esfuerzo por incorporar la educación técnica en el Departamento de Santander (1887-1937), financiados por la Universidad Industrial de Santander (UIS).

2. Profesor Titular de la Universidad Industrial de Santander. Doctor en Historia. Director del Grupo de Investigación Políticas, Sociabilidades y Representaciones Histórico-Educativas. E-mail: tarazona20@ gmail.com 


\title{
UNIVERSITY: FROM HUMAN CAPITAL TO A BUSINESS MODEL IN HIGHER EDUCATION
}

\begin{abstract}
This article is a reflection on the role of universities in society and their changing configuration into a business. The American university model, prevailing in the sixties and seventies, based on the technical and scientific knowledge, is being replaced by a neoliberal concept that has made higher education a business unit from the logic of the market. In this debate, the university has been considered from two perspectives: on its role as a business or as an institution that promotes knowledge. The latter role has posed questions on higher educational issues and local and national approaches.
\end{abstract}

Key words: University, research, knowledge, enterprise, human capital.

\section{UNIVERSIDADE: DE CAPITAL HUMANO A UM MODELO DE EMPRESA NA EDUCAÇÃO SUPERIOR}

\begin{abstract}
RESUMO
Este artigo é uma reflexão do papel da Universidade na sociedade e suas mudanças na configuración, até se tornar em uma empresa. 0 modelo da universidade norteamericana, prevalecente durante os anos sessenta e setenta, fundamentado na técnica e o conhecimento científico, o está substituindo um conceito neoliberal que fez da Educação Superior uma unidade empresarial partindo das lógicas do mercado. Neste debate, a universidade surgiu a partir de dois lados: em sua função de empresa ou de conhecimento, o que levanta a questão pelos temas educativos do superior, sem resolução do continente, e as abordagens locais e nacionais.
\end{abstract}

Palavras chave: Universidade, investigação, conhecimento, empresa, capital humano. 


\section{INTRODUCCIÓN}

La instrucción y el aprendizaje no son un espacio neutral, donde se difuminan el poder, la historia y el contexto social. Todo lo contrario, el campo educativo es un entramado de prácticas hegemónicas ${ }^{3}$. Esto quiere decir que la educación trasciende el espacio de las teorías y modelos para ubicarse en la producción y construcción de significados de la cultura y en las relaciones de la política y de la cotidianidad ${ }^{4}$. La práctica educativa es, sobre todo, una práctica política, porque su campo de acción no sólo se limita a las aulas ${ }^{5}$.

En América Latina, los fundamentos de la educación se implementaron sobre una racionalidad instrumental con pretensiones universales y sin preguntarse por el tipo de formación social e histórica y los regímenes políticos de los Estados nacionales. En el caso de la universidad, ya desde el siglo XIX, la reforma de Andrés Bello proponía la asimilación crítica del saber europeo como una forma de reconocer las limitaciones disciplinarias y tecnológicas de los Estados nacionales todavía en formación. Sin embargo, en el siglo XIX, la sustitución de la universidad colonial por la napoleónica, derivó hacia un énfasis profesionalista ${ }^{6}$.

De una universidad colonial escolástica, memorística y sólo para peninsulares, clérigos, criollos e hijos de caciques principales, cuya misión fundamental era salvar al hombre, se pasó a una universidad profesionalista, tutelada por el Estado, cuya misión era formar los administradores de la burocracia estatal y suplir las necesidades sociales. Pese a que la universidad colonial fue excluyente, guardó una unidad como corporación de maestros y estudiantes (por sus relaciones con el modelo de Bolonia y Salamanca), lo cual le permitió regentarse por el autogobierno de claustros y dar alguna participación a los estudiantes en el momento de elegir a sus profesores.

La universidad republicana, en cambio, por su énfasis profesionalista, se concibió como una suma de Facultades y, al igual que la universidad colonial, carente de investigación, sin dejar der ser también excluyente por su carácter elitista. Ahora bien, si se compara la universidad colonial con la republicana, hubo más unidad de criterio en la primera que en la segunda ${ }^{7}$.

3. Peter McLaren. Teoría crítica y significado de la esperanza. En: Henry A. Giroux. Los profesores como intelectuales: hacia una pedagogía crítica del aprendizaje (Barcelona: Paidós, 1997): 11-27.

4. Humberto Maturana. Trasformación en la convivencia (Santiago de Chile: Dolmen Ediciones, 2002).

5. McLaren, Teoría crítica y significado de la esperanza.

6. Álvaro Acevedo Tarazona y Gabriel David Samacá Alonso. De la Reforma de Córdoba al Cordobazo: la universidad como escenario de luchas por la democracia argentina 1918-1969 y su impacto en Colombia. En: Memorias. Vol. 15 (2011): 170-195.

7. Carlos Tunnermann Bernheim. Historia de la Universidad en América Latina: de la época colonial a la Reforma de Córdoba (Caracas: IESALC/UNESCO, 1999). 
En este largo tránsito de la Colonia a la República, y luego hasta el siglo XX, sólo hasta los años ochenta de esta centuria, las universidades latinoamericanas se impusieron el desafío de formar tanto para las competencias profesionales como para la investigación. De manera que la universidad, en América Latina, sólo hasta hace muy pocos decenios, hizo de la investigación un propósito fundamental. No obstante, este fin, hasta el momento, no ha sido exitoso por no ajustarse a la realidad de sus contextos, por su masificación y por alinearse con los requerimientos del mercado laboral.

Hoy, la universidad sigue formando más para el ejercicio profesional que para la investigación científica. Y, aunque se presentan reconocidos avances en este último propósito, la investigación continúa siendo dispersa, individualista y específica. Hoy, la universidad sigue reclamando la construcción de una nueva cultura académica que valore la investigación científica, trasfiera el conocimiento como resultado de la investigación y se preocupe por la valoración del conocimiento adquirido ${ }^{8}$.

\section{UNIVERSIDAD: ENTRE EL CAPITAL HUMANO Y LA COMERCIALIZACIÓN DE LA EDUCACIÓN}

Tras una contradictoria implementación, en algunos países de América Latina, del modelo universitario norteamericano, durante los años sesenta y setenta de la pasada centuria, $\mathrm{y}$, a partir de las reformas económicas en todas las esferas del Estado, ejecutadas durante los decenios ochenta y noventa, la Educación Superior, en América Latina, entró en las lógicas del neoliberalismo. Además de los recortes en el gasto estatal para el funcionamiento y expansión de las universidades públicas, dichas lógicas correspondieron a la implementación de la teoría del capital humano, que hasta el día de hoy considera a la inversión en educación bajo la relación consumo-inversión, lo que, en otras palabras, significa preguntarse por la tasa de retorno (rentabilidad) a la inversión en educación ${ }^{9}$. Formar capital humano significa realizar inversiones en la formación profesional de un individuo, porque se lo considera una especie de idoneidad-máquina que va a producir rentas, o va a remunerarse con un ingreso ${ }^{\mathbf{1 0}}$. Las adecuaciones educativas se orientan más hacia un cálculo económico deliberado que hacia un asunto vocacional o formativo, y persiguen intereses que supuestamente beneficiarán a la sociedad en su conjunto.

8. Gabriel Misas Arango. La Educación Superior en Colombia: análisis y estrategias para su desarrollo (Bogotá: Universidad Nacional de Colombia, 2004).

9. Carlos Alberto Torres. Grandezas y miserias de la educación latinoamericana del siglo XX, en: Carlos Alberto Torres (comp.) Paulo Freire y la agenda de la educación latinoamericana siglo XXI (Buenos Aires: CLACSO, 2001), http://www.clacso.org (20 de enero de 2010).

10. Michel Foucault. Nacimiento de la biopolítica (México: Fondo de Cultura Económica, 2007). 
Actualmente, las inversiones en el sector educativo se cuantifican mediante diversos indicadores para la maximización de utilidades financieras. La educación universitaria pasa a cuantificarse, planearse y controlarse en términos de producción y eficiencia, como si fuera una empresa más para el mercado y la rentabilidad. Para alcanzar estas metas, se recurre a los imaginarios constituyentes de la calidad y la innovación ${ }^{11}$ : el primero se adquiere mediante una eficacia, que orienta las acciones a la adquisición de conocimientos, capacidades y habilidades mediante la maximización de las acciones; el segundo se alcanza por intermedio de la eficiencia; léase, también, adaptación e innovación, es decir, mediante el descubrimiento de nuevas formas de productividad, especialmente tecnológicas. La formación con estos parámetros ha establecido un modelo profesional universitario sin humanismo y sin el ethos científico requerido para hacer del conocimiento un fin en sí mismo, y no sólo un medio para la renta y la movilidad social ${ }^{12}$.

La lógica del mercado, en la universidad, se constituyó en un hecho educativo hasta vaciar los objetivos de las preocupaciones humanistas o culturales. En medio de ello, las universidades han sido copartícipes de una objetivación instrumental, tanto del conocimiento como de los individuos educables. La idea de una universidad al servicio de la sociedad del conocimiento se ha perdido. La universidad actual ha olvidado su función social para producir un conocimiento útil y comercializable, de manera que los para qué y para quién del conocimiento quedan casi siempre fuera de debate, lo que limita el trabajo científico a los cómo, a construir conocimiento operativo, instrumental, que busca medios para atender fines, que no se discuten ${ }^{13}$.

En 1995, el Banco Mundial impulsó una trasformación de la Educación Superior, de acuerdo con las estrategias del libre mercado y sugirió la privatización de las universidades. Contraria a esta posición, la UNESCO, en 1995 y 1998, definió claramente a la educación como un bien social, para impulsar el desarrollo humano sostenible, considerado como "no sólo un perfeccionamiento en el sentido empresarial de la expresión, sino en un sentido más amplio, con la educación y la formación como elementos esenciales"14.

Para el Banco Mundial, es imperativo que las universidades hicieran reformas presupuestales; para tal efecto, señala "la conveniencia de finalizar con el modelo

11. María Isabel Lafuente Guantes. La educación universitaria hoy: desde la convergencia entre calidad e imaginarios sociales. En: Revista de Ciencias Humanas. No. 36 (2007): 127-145.

12. Álvaro Acevedo Tarazona. Modernización, conflicto y violencia en la universidad en Colombia (AUDESA 1953-1984) (Bucaramanga: UIS, 2004).

13. Gabriel Kaplún. Indisciplinar la universidad (Quito: Universidad Andina Simón Bolívar, 2004).

14. Ángel Díaz Barriga. Contexto nacional y políticas públicas para la educación superior en México, 19501995. En: Hugo Casanova y Roberto Rodríguez. Universidad contemporánea: política y gobierno (México: Porrúa, 1999), Tomo II, 384. 
del presupuesto negociado en el que el gobierno financia a las instituciones sobre la base de criterios tradicionales, como la matrícula y el prestigio". Se indica que, por el contrario, "se debe dar paso al modelo de presupuesto por rendimiento, cuyo criterio base establece que la financiación pública, que permanezca, debe estar sujeta a la obtención de resultados comprobables valorados en función de indicadores establecidos de acuerdo con los resultados obtenidos por el consumidor"15.

El enfoque empresarial de la educación promueve un debate entre quienes se oponen a que se tratase como mercancía y quienes sostienen que "el comercio educativo está vigente y aporta beneficios y posibilidades, siempre que se regule de manera correcta"16. En esta lógica empresarial, la universidad podría innovar sus modelos de docencia, investigación y extensión con el propósito de ampliar la oferta de servicios educativos de calidad. Para esta misma tendencia, la educación, más que un problema, entraría en sintonía con la globalización para atender un comportamiento por ahora irreversible: el triunfo de la economía neoliberal.

\section{LA ACTUALIDAD DE LA EDUCACIÓN SUPERIOR EN AMÉRICA LATINA}

El siglo XX tuvo una especial significación para el campo educativo. De esta centuria fueron propios los procesos estatales de incentivo a la formación escolar temprana y ampliación de la cobertura en los niveles secundarios.

La masificación de la educación pública generó, en el mediano plazo, un incremento de oportunidades para el acceso a la formación técnica y profesional. Sectores sociales de América Latina antes excluidos de la educación se vincularon a esta tendencia formativa; hoy, esta tendencia continúa teniendo un gran impacto en un intento por asimilar el ideal ilustrado de progreso ${ }^{17}$, lo que ha tenido especiales repercusiones en las universidades públicas del subcontinente, cuyas finanzas entraron en crisis ante un crecimiento absoluto de la demanda educativa que no tuvo correspondencia equitativa en las asignaciones presupuestales del Estado, más todavía con la implantación del modelo neoliberal de racionalización del gasto público a partir de los años ochenta y noventa del siglo pasado.

Ante tal problemática, los centros privados de Educación Superior surgieron como la principal alternativa para absorber la creciente masa de individuos interesados en acceder a la formación universitaria, que no podían ingresar al

\footnotetext{
15. Diana Soto. Criterios comunes para el desarrollo de una educación universitaria global: una propuesta latinoamericana. En: María Isabel Lafuente Guantes. ¿Hacia dónde va la educación universitaria americana y europea? Historia, temas y problemas de la universidad (León: Universidad de León, 2006), 35.

16. Manuel Cebrián de la Serna. Recursos tecnológicos para los procesos de enseñanza y aprendizaje (Málaga: ICE/Universidad de Málaga, 1998), 9.

17. Torres, Grandezas y miserias de la educación latinoamericana del siglo XX.
} 
sistema público universitario ${ }^{18}$. Se asistió, entonces, a un proceso de democratización de la educación, mas no de la calidad educativa.

La rápida multiplicación de las universidades privadas implicó la consolidación de todo un mercado educativo particular al mando de nuevos actores: empresarios de la educación que han sabido capitalizar a su favor las realidades del campo educativo, en un escenario de ascenso social y satisfacción de un mercado laboral cada vez más competitivo.

En los últimos veinte años, la Educación Superior, en América Latina, se ha orientado por dos tendencias: la consolidación, por una parte, de macro-universidades públicas y, por la otra, una inusitada aparición de universidades privadas ${ }^{19}$. Al ritmo de esta profunda dualidad, los crecientes sectores de población interesados en adquirir una formación profesional afrontan una preocupante paradoja. Si no logran acceder a una universidad pública reconocida o no cuentan con los recursos para costearse estudios en alguna universidad privada de calidad, se ven casi que forzados a formarse en centros de Educación Superior privados, con una baja calidad académica.

Como resultado de la instrumentalización del conocimiento y la peculiar inserción de la Educación Superior en las lógicas del mercado, se ha presentado una distorsión del interés científico y la actividad investigativa. La implementación de estándares de excelencia académica trata de vincular la formación tecnológica con los sectores productivos (inserción técnica en la economía del mundo), pero no se preguntan por el cambio de un modelo educativo en un mundo con problemas de deterioro del medio ambiente, conflictos culturales y la brecha cada vez más abismal entre ricos y pobres, lo que significa pensar en una educación interrelacionada con el contexto social y la investigación, pero en América Latina la escuela y la universidad pública cada vez pierden estos escenarios al privilegiarse la formación por competencias y enfoques profesionales especializados.

En la configuración de esta realidad, ha tenido un papel preponderante la asimilación, en las universidades latinoamericanas, de los principios del neoliberalismo, en especial la vertiente norteamericana del capital humano, tendencia que proviene de una reinterpretación crítica del papel del trabajo en el análisis económico clásico y se constituye en uno de los pilares fundamentales de las propuestas de innovación como factor fundamental para el desarrollo económico de una sociedad ${ }^{20}$. Con este ideario, se han constituido, en los últimos años, teorías y adecuaciones sobre una racionalidad instrumental propia de una cultura racional

18. Rollin Kent. Los temas críticos de la Educación Superior de América Latina (México: Fondo de Cultura Económica, 2002).

19. Kaplún, Indisciplinar la universidad.

20. Foucault, Nacimiento de la biopolítica. 
calculadora de medios para fines que promueve la satisfacción de preferencias, se obsesiona por el control y aspira a un ideal universal de educación, como si a todos los seres humanos se les pudiese despojar de los símbolos y tradiciones.

Junto a esta idea del capital humano, se han establecido también los parámetros sobre quién financia y cómo se paga la investigación en América Latina. El financiamiento de las empresas, propio también ahora de una visión corporativa de universidad, gobernada en sus propósitos por el sector privado, ha tenido un significativo auge en el tiempo reciente. Mediante esta modalidad, los empresarios definen los campos y propósitos de la investigación científica universitaria de acuerdo con sus intereses peculiares y con la complacencia de las entidades gubernamentales. Los publicitados Comités Universidad-Empresa-Estado, tan en boga actualmente en América Latina, reproducen fielmente este modelo. Otra vía para el desarrollo investigativo es acceder, por concurso, a fondos públicos. En este caso, las prioridades se acuerdan entre el Estado y la administración universitaria, para configurar un escenario en el que los investigadores se ven sometidos a la lógica de los proyectos (sometimiento de la investigación a la burocracia administrativa) como única alternativa para el desarrollo de sus labores ${ }^{21}$.

Los principios que guían las actividades de investigación científica en las universidades no corresponden tanto a un avance articulado del conocimiento, como sí a las posibilidades de rentabilidad para los inversionistas privados de los mecanismos o procedimientos que puedan generar las investigaciones. De este modo, y como se ha señalado previamente, se ha configurado un paradójico contexto de orientación en las inversiones para la Educación Superior, en los Estados latinoamericanos, en el que predomina una preocupante mercantilización del conocimiento.

Tabla 1. Proyección del crecimiento de la universidad a nivel mundial. Periodo 1991-2025.

\begin{tabular}{|l|c|c|c|c|}
\hline Matrícula Año & 1991 & 2000 & 2015 & 2025 \\
\hline En millones & 65 & 79 & 97 & 100 \\
\hline
\end{tabular}

Fuente: UNESCO. Tendencias de la Educación Superior. 1995.

En el espectro de esta realidad paradójica, es posible comprender mejor las tendencias actuales de la universidad de América Latina, no sólo en el contexto subcontinental propio, sino también en el contraste con las realidades globales contemporáneas. En términos absolutos (Tabla 1), se identifica con claridad el

21. Kaplún, Indisciplinar la universidad. 
progresivo crecimiento del número de estudiantes universitarios a nivel mundial. Al desagregar estas cifras a nivel latinoamericano, los positivos registros de las matrículas y la cobertura en Educación Superior (Tabla 2) confirman esta tendencia. Dicha expansión se encuentra matizada por el surgimiento de instituciones de Educación Superior de baja calidad y una mercantilización del conocimiento que ha dificultado la articulación del saber científico de las universidades en beneficio de las sociedades que sustentan su funcionamiento.

Tabla 2. Crecimiento de la Educación Superior en América Latina. Periodo 1970-2005.

\begin{tabular}{|c|c|c|c|c|}
\hline Año & Matrícula & $\begin{array}{c}\text { Aumento quinque- } \\
\text { nal de matrícula } \\
\text { absoluta (\%) }\end{array}$ & $\begin{array}{c}\text { Cobertura } \\
\text { (población: 20- } \\
\text { 24 años) }\end{array}$ & $\begin{array}{c}\text { Incremento quin- } \\
\text { quenal. Tasa de } \\
\text { cobertura (\%) }\end{array}$ \\
\hline 1970 & 1.646 .308 & & 7.03 & \\
\hline 1975 & 3.450 .526 & 109.6 & 12.27 & 74.54 \\
\hline 1980 & 4.622 .364 & 34.0 & 14.22 & 15.89 \\
\hline 1985 & 5.936 .846 & 28.4 & 15.77 & 10.90 \\
\hline 1990 & 6.703 .442 & 12.9 & 16.45 & 4.31 \\
\hline 1995 & 8.146 .843 & 21.6 & 18.43 & 12.03 \\
\hline 2000 & 11.269 .127 & 38.3 & 23.40 & 26.06 \\
\hline 2005 & 15.932 .105 & 41.37 & 31.77 & 35.76 \\
\hline
\end{tabular}

Fuente: Claudio Rama. Los estudios de la masificación de la Educación Superior en América Latina y Sistemas de Estadísticas Nacionales (IESALC/CEPAL/IUS, 2009).

La masificación de la universidad en América Latina crece a tasas superiores al 7\% interanual, tendencia que incrementa el número de egresados y promueve entre ellos una mayor competencia en los mercados laborales. Ha sido de tal magnitud la tendencia de la masificación de la Educación Superior en América Latina, entre 1970 y 2005 , que ha pasado de $7.03 \%$ a $31.77 \%$, esto es, de 1.6 millones de estudiantes, en 1970, a 15.9 millones, en el año $2005^{22}$.

La creación de posgrados con fundamentos investigativos fue un distintivo del modelo universitario norteamericano, al igual que su relación de continuidad con las academias profesionales y los estudios de pregrado. Si bien el modelo universitario norteamericano se convirtió en garante del conocimiento científico mediante principios organizados en la economía de mercado, la asociación democrática y la burocracia administrativa con propósitos de planificación y

22. Jamil Salmi. Educación superior: enfrentando los retos del Siglo XXI, www.wordbank. org/education/ tertiar (25 de septiembre de 2008). 
eficiencia, la universidad en el continente latinoamericano - con base en este modelo universitario descrito- se burocratizó y estableció una baja calidad en los estudios básicos; los departamentos se fragmentaron, las responsabilidades académicas se trastornaron y la investigación se desarrolló, en estricto, en los posgrados.

Cabe destacar que este modelo educativo fue exitoso en Estados Unidos porque se ajustó a la necesidad norteamericana de consolidar una economía planificada sostenida en el individualismo económico y la propia iniciativa. También fue exitoso, contrario a lo que aconteció en América Latina, porque las innovaciones produjeron ruptura con residuos de fases históricas pasadas, lo que no sucedió en el escenario latinoamericano, en el que se crearon híbridos, como el modelo profesionalizante republicano de universidad. En estas consideraciones, se puede matizar el sorprendente crecimiento de la formación posgraduada en América Latina en las últimas décadas (Tabla 3).

Tabla 3. Universidad latinoamericana: Estudiantes de posgrados. Periodo 1995-2006.

\begin{tabular}{|l|c|c|c|c|c|c|}
\hline \multirow{2}{*}{ Nivel } & \multicolumn{2}{|c|}{1995} & \multicolumn{2}{c|}{2002} & \multicolumn{2}{c|}{2006} \\
\cline { 2 - 7 } & Cantidad & $\%$ & Cantidad & $\%$ & Cantidad & $\%$ \\
\hline Especialización & 61.311 & 33 & 187.319 & 35 & 322.188 & 35 \\
\hline Maestría & 101.968 & 55 & 278.303 & 52 & 512.077 & 55 \\
\hline Doctorado & 22.094 & 12 & 69.576 & 13 & 92.755 & 10 \\
\hline Total & 185.373 & 100 & 535.198 & 100 & 927.020 & 100 \\
\hline Incremento entre períodos & & & 349.825 & 65 & 391.822 & 73 \\
\hline
\end{tabular}

Fuente: Francisco López Segrera. Cátedra Educación Superior y Sociedad (Cali: Universidad Santiago de Cali, 2006); Claudio Rama. La tercera reforma de la educación superior en América Latina y el Caribe: masificación, regulaciones e internacionalización. En: IESALC. Informe sobre Educación Superior en América Latina y el Caribe 2000-2005: la metamorfosis de la Educación Superior (Caracas: Metrópolis, 2006).

Ante la creciente trasformación de los mercados laborales, paralelo a la ampliación de la cobertura universitaria, se ha presentado un impulso a la educación para toda la vida y a la educación posgraduada. El decenio de los noventa se caracterizó por un crecimiento exponencial de los posgrados. Aunque a primera vista se observen resultados favorables, las actividades de posgrado en América Latina implican un fenómeno de autofinanciación de las universidades públicas, en contraste con un preocupante detrimento de los programas de pregrado. Los mejores recursos y posibilidades se garantizan para aquellos que puedan pagar, lo que demuestra que la vinculación de la educación al trabajo, unida a la medición de la calidad académica por intermedio de estándares internacionales, hoy 
es más un instrumento de control político que una alternativa de mejoramiento educativo.

La educación ha dejado de ser un bien público para constituirse en una orientación competitiva hacia el mercado y la transnacionalización de la economía ${ }^{23}$. La apertura económica y la globalización niegan el carácter de la educación como un derecho social, ahondan la descentralización financiera y administrativa del sistema educativo y centralizan la evaluación y las orientaciones curriculares con el concepto de calidad. De esta manera, el Estado, por un lado, desatiende su responsabilidad financiera; del otro, regula las políticas curriculares y de aprendizaje y entiende la evaluación como una medida y control de rendimiento, y no como un proceso que diagnostica la realidad en sus múltiples dimensiones, para operar sobre ella.

Tabla 4. Doctorados en América Latina. Periodo 2003-2004.

\begin{tabular}{|l|c|c|c|}
\hline \multicolumn{1}{|c|}{ País } & Doctorados & Porcentaje & Universidades \\
\hline Brasil & 1.056 & $48.3 \%$ & 52 \\
\hline México & 406 & $18.5 \%$ & 80 \\
\hline Argentina & 291 & $13.3 \%$ & $\mathrm{~s} / \mathrm{d}$ \\
\hline Chile & 103 & $4.7 \%$ & 10 \\
\hline Cuba & 95 & $4.3 \%$ & 12 \\
\hline Perú & 91 & $4.2 \%$ & 17 \\
\hline Colombia & 56 & $2.6 \%$ & 17 \\
\hline Venezuela & 48 & $2.2 \%$ & 7 \\
\hline Uruguay & 11 & $0.5 \%$ & 1 \\
\hline Ecuador & 9 & $0.4 \%$ & 4 \\
\hline Bolivia & 8 & $0.4 \%$ & 5 \\
\hline Paraguay & 7 & $0.3 \%$ & 4 \\
\hline Panamá & 5 & $0.2 \%$ & 4 \\
\hline TOTALES & $\mathbf{2 . 1 8 8}$ & $\mathbf{1 0 0} \%$ & $\mathbf{2 1 3}$ \\
\hline
\end{tabular}

Fuente: INSTITUTO INTERNACIONAL PARA LA EDUCACIÓN SUPERIOR DE AMÉRICA LATINA Y EL CARIBE (IESALC). Informe sobre la Educación Superior de América Latina y el Caribe 2000-2005: la metamorfosis de la Educación Superior (Caracas: Metrópolis, 2006).

23. López Segrera, Cátedra Educación Superior y Sociedad. 
El caso de la formación doctoral en las universidades latinoamericanas no dista de la problemática general de la formación de los posgrados: más de lo mismo con los mismos resultados, porque no surgen de la actividad de la investigación sino como parte de una necesidad burocrática; como producto de ello, se evidencia un desplazamiento del talento y de la calidad académica y se instrumentaliza su función: consumo, producción, productividad, difusión, impacto. Muestra de ello es que, entre 1994 y 2000, la tasa de incremento en Latinoamérica alcanzó el 31\% interanual; Brasil y México, con más de 8.000 doctores año, seguidos por Argentina y Venezuela.

Hoy no se puede pasar por alto que la tendencia en la formación de investigadores en niveles de maestría y doctorado, en América Latina, aumenta por las exigencias de un medio externo cada vez más rígido respecto a la calidad. Mientras a las especializaciones van los profesionales que necesitan profundizar en sus disciplinas y saberes, a las maestrías van generalmente expertos vinculados con los sectores académicos e investigativos.

\section{COBERTURA, FORMACIÓN POSGRADUADA Y CALIDAD}

Hoy, en América Latina, se acrecienta la confrontación entre universidad privada y pública, en un escenario de globalización en el que se rebasan las fronteras nacionales, hasta que el Estado pierde capacidad en la construcción de un proyecto de universidad. La apertura económica y la globalización han negado el carácter de la educación como un derecho social, han profundizado la descentralización financiera y administrativa del sistema educativo y centralizado la evaluación y las orientaciones curriculares con el concepto de calidad.

En Latinoamérica, las grandes universidades públicas tienen programas de investigación asociados a posgrados. En las universidades privadas, esta relación es muy baja, a lo que se suma la mercantilización de la oferta. La movilidad internacional también es muy poca, por la incapacidad de generar oferta en el nivel doctoral. José Joaquín Brunner señala que, en la actualidad, hay tres tipos de universidad: de investigación (menos del 3\%), con investigación (quizá un $7 \%$ ) y puramente docente (más del $90 \%$ ), razón por la cual se considera a la universidad de corte profesionalizante.

La calidad de la educación adquiere un valor determinante para los Estados nacionales, por la necesidad que tienen de regular las instituciones de Educación Superior, lo que se debe a que se ha erosionado la confianza en este tipo de educación por el crecimiento de las instituciones y de la matrícula, por la diversificación de programas e instituciones y por las ofertas trasnacionales.

Para la UNESCO, la calidad de la Educación Superior es un concepto pluridimensional, lo que quiere decir que debe incluir todas las funciones sustantivas que le son propias y que su institucionalidad está en permanente autoevaluación 
por intermedio de marcos comparativos de calidad, especialmente reconocidos en el plano internacional, sin desconocer la diversidad.

En los últimos años, se observa en las universidades un interés vital por la búsqueda de calidad. Lo cierto es que dicho concepto proviene de la empresa. Tampoco se puede desconocer que el concepto ha tenido trasformaciones, desde el enfoque cuantitativo hasta aquellas concepciones que se preocupan por satisfacer las demandas de los usuarios. Tanto el usuario (estudiante) como el productor (universidad) son las piezas claves de la calidad: el primero, como destinatario, y el segundo, como el oferente de la demanda. En las dos últimas décadas, se crearon, en América Latina, numerosas instituciones de Educación Superior para satisfacer la demanda, la mayoría de ellas privadas y sin criterios de calidad.

La rendición de cuentas es un requisito básico en la relación universidad/ sociedad, más aún por el carácter de bien público que tiene la universidad. Se trata, entonces, de no negar las certificaciones y acreditaciones, pero no sólo ubicarse en la rayana medición de resultados por indicadores. La pertinencia, equidad, eficiencia, trasparencia y, sobre todo, la condición de servicio público de las instituciones de Educación Superior van más allá de los baremos por resultados.

\section{CONCLUSIONES}

Si la educación es la apuesta por el acceso a prácticas de cultura, convivencia, sociabilidad y dignidad, su ejercicio debe comprender la dimensión histórica de tales fines exitosos o fallidos en América Latina y, especialmente, reconocer los múltiples problemas y vertientes del debate universitario frente a las políticas y modernizaciones educativas en el continente.

Como un hecho social e histórico que es la educación, en el que se movilizan discursos, prácticas y relaciones de sentido y de poder, es tarea del educador y de los intelectuales reconocer las luchas y tensiones ideológicas y políticas del proceso formativo.

La universidad, en la segunda mitad del siglo XX, no se puede comprender sin una revisión del proyecto educativo modernizador de los años sesenta y setenta. La apuesta que hicieron los gobiernos y elites económicas por alcanzar a las sociedades desarrolladas fue el telón de fondo para imaginar una universidad al servicio del progreso material de las sociedades "subdesarrolladas". No obstante, hubo conciencia de la necesidad de adecuar la Educación Superior a los propósitos económicos del desarrollo capitalista. Sin embargo, ese anhelo de modernización de la Educación Superior como condición necesaria para alcanzar el desarrollo material de la nación llevó inevitablemente a una mercantilización del conocimiento, que deja a un lado la formación del capital humano y desarrolla patrones de competencia que aún están por revisar. 


\section{REFERENCIAS BIBLIOGRÁFICAS}

Acevedo Tarazona, Álvaro. Modernización, conflicto y violencia en la universidad en Colombia (AUDESA 19531984). Bucaramanga: UIS, 2004.

Reforma y reformismo universitario en la Universidad de América Latina y el Caribe. De la propuesta de reforma de Andrés Bello al instrumentalismo reformista de los años sesentas y setentas del siglo XX. En: Revista Historia y Espacio. No. 30 (2008): 61-81.

Acevedo Tarazona, Álvaro y Gabriel David Samacá Alonso. De la Reforma de Córdoba al Cordobazo: la universidad como escenario de luchas por la democracia argentina 1918-1969 y su impacto en Colombia. En: Memorias. Vol. 15 (2011): 170-195.

Asociación Colombiana de Universidades y Fondo Universitario Nacional. Plan Básico de la Educación Superior en Colombia: documentos. Vol. 1. Bogotá: Imprenta Nacional, 1969.

Atcon, Rudolph. La universidad latinoamericana: clave para un enfoque conjunto del desarrollo coordinado social, económico y educativo en América Latina. En: Revista Eco. Tomo VII (1963), http://www.scribd.com/ full/52353452?access_key=key-1wyqusat9kylnm8x2y34 (27 de enero de 2014).

Briceño, Rosa. University reform, social conflict, and the intellectuals: the case of the National University of Colombia. Palo Alto, California: Universidad de Stanford, 1988 (Tesis de Doctorado en Filosofía).

Brunner, José Joaquín. Educación en América Latina durante la década de 1980: la economía política de los sistemas. En: Kent, Rollin. Los temas críticos de la Educación Superior de América Latina. México: Fondo de Cultura Económica, 2002.

. Aseguramiento de la calidad y nuevas demandas sobre la Educación Superior en América Latina. Bogotá: Consejo Nacional de Acreditación, 2003.

Cebrián de la Serna, Manuel. Recursos tecnológicos para los procesos de enseñanza y aprendizaje. Málaga: ICE/Universidad de Málaga, 1998.

Díaz Barriga, Ángel. Contexto nacional y políticas públicas para la educación superior en México, 1950-1995. En: Casanova, Hugo y Roberto Rodríguez. Universidad contemporánea: política y gobierno. Tomo II. México: Porrúa, 1999.

Fernández, Norberto. La evaluación y la acreditación de la calidad: situación, tendencias y perspectivas. En: Instituto Internacional para la Educación Superior de América Latina y el Caribe (IEASALC). Informe sobre la educación superior en América Latina y el Caribe, 2000-2005: la metamorfosis de la educación superior. Caracas: Metrópolis, 2006.

Foucault, Michel. Nietzsche, la genealogía, la historia. Valencia: Pretextos, 1997.

. Nacimiento de la biopolítica. México: Fondo de Cultura Económica, 2007.

García, Antonio. La crisis de la universidad: la universidad en el proceso de la sociedad colombiana. Bogotá: Plaza y Janés, 1985.

Hernández, Isabel. El ethos investigativo del docente de la Universidad Cooperativa de Colombia, vinculado a esta función sustantiva en las seccionales de Bogotá, Medellín y Pasto. Pasto: Universidad de Nariño/RUDECOLOMBIA, 2010 (Tesis de Doctorado en Ciencias de la Educación).

Instituto Internacional para la Educación Superior de América Latina y el Caribe (IESALC). Informe sobre la educación superior de América Latina y el Caribe 2000-2005: la metamorfosis de la educación superior. Caracas: Metrópolis, 2006.

Jiménez, Absalón y Helwar Figueroa. Políticas educativas en la Educación Superior: 1925-1992. En: Revista Colombiana de Educación. No. 38 (2000): 181-200.

Kaplún, Gabriel. Indisciplinar la universidad. Quito: Universidad Andina Simón Bolívar, 2004. 
Kent, Rollin. Los temas críticos de la Educación Superior de América Latina. México: Fondo de Cultura Económica, 2002.

Lafuente Guantes, María Isabel. La educación universitaria hoy: desde la convergencia entre calidad e imaginarios sociales. En: Revista de Ciencias Humanas. No. 36 (2007): 127-145.

Le Bot, Yvon. Educación e ideología en Colombia. Bogotá: La Carreta, 1985.

López Segrera, Francisco. Cátedra Educación Superior y Sociedad. Cali: Universidad Santiago de Cali, 2006.

. América Latina y el Caribe: globalización y Educación Superior. En: López Segrera, Francisco. Escenarios mundiales de la educación superior. Análisis global y estudios de casos. Buenos Aires: CLACSO, 2006, http://www.riseu. unam.mx (26 de noviembre de 2007).

Maturana, Humberto. Trasformación en la convivencia. Santiago de Chile: Dolmen Ediciones, 2002.

McLaren, Peter. Teoría crítica y significado de la esperanza. En: Giroux, Henry A. Los profesores como intelectuales: hacia una pedagogía crítica del aprendizaje. Barcelona: Paidós, 1997: 11-27.

Misas Arango, Gabriel. La Educación Superior en Colombia: análisis y estrategias para su desarrollo. Bogotá: Universidad Nacional de Colombia, 2004.

Molina Rodríguez, Carlos Alberto. FUN-ASCÚN en la historia del sistema universitario colombiano, 1958-1968. Bucaramanga: Universidad Industrial de Santander, 2013.

Ordoñez Burbano, Luis Aurelio. Universidad del Valle 60 años, 1945-2005: atando cabos en clave de memoria. Cali: Universidad del Valle, 2007.

Rama, Claudio. La tercera reforma de la Educación Superior en América Latina y el Caribe: masificación, regulaciones e internacionalización. En: Instituto Internacional para la Educación Superior de América Latina y el Caribe (IESALC). Informe sobre educación superior en América Latina y el Caribe 2000-2005: la metamorfosis de la Educación Superior. Caracas: Metrópolis, 2006.

Los estudios de la masificación de la Educación Superior en América Latina y Sistemas de Estadísticas Nacionales. IESALC-CEPAL-IUS, 2009.

Salmi, Jamil. Educación Superior: enfrentando los retos del Siglo XXI, www.wordbank. org/education/tertiar (25 de septiembre de 2008).

Samoilovich, Daniel. Senderos de innovación: repensando el gobierno de las universidades públicas en América Latina. En: Gazzola, Ana Lúcia y Axel Didriksson. Tendencias de la Educación Superior en América Latina y el Caribe. Caracas: IEASALC/UNESCO, 2008.

Sánchez, Alejandro y Mónica Zuleta. La batalla por el pensamiento propio en Colombia. En: Nómadas. No. 27 (2007): 113-135.

Soto, Diana. Criterios comunes para el desarrollo de una educación universitaria global: una propuesta latinoamericana. En: Lafuente Guantes, María Isabel. ¿Hacia dónde va la educación universitaria americana y europea? Historia, temas y problemas de la universidad. León: Universidad de León, 2006.

Torres, Carlos Alberto. Grandezas y miserias de la educación latinoamericana del siglo XX. En: Puigross, Adriana, et al. Paulo Freire y la agenda de la educación latinoamericana en el siglo XXI. Buenos Aires: CLACSO, 2001, http://www.clacso.org. Fecha de consulta: 20 de enero de 2010.

Tunnermann Bernheim, Carlos. Historia de la Universidad en América Latina: de la época colonial a la Reforma de Córdoba. Caracas: IESALC/UNESCO, 1999.

Universidad Nacional de Colombia. Plan Básico de la Educación Superior: análisis crítico. Bogotá: Universidad Nacional de Colombia, 1969. 